Santos, L., Ramírez, E. (2016). De la contradicción entre la crisis de las profesiones agropecuarias y el potencial agropecuario colombiano. Revista Lebret, 8. Bucaramanga, Colombia: Universidad Santo Tomás, pp. 293 - 308. ISSN: 2145-5996

\title{
De la contradicción entre la crisis de las profesiones agropecuarias y el potencial agropecuario colombiano*
}

\section{The contradiction between the crisis of agricultural professions and the colombian agricultural potential}

Luis Eduardo Santos Padilla

Edwar D. Ramírez Castellanos ${ }^{2}$

\begin{abstract}
Resumen
El presente artículo busca poner en común y ampliar la discusión sobre la contradicción entre la crisis en número de estudiantes que ingresan a las facultades agropecuarias en Colombia, a partir de la última década del siglo XX, y el potencial para la producción de alimentos, expresado por organismos como FAO, OCDE y Mesa de Conversaciones de la Habana, soportada en indicadores como disponibilidad de tierras, abundancia de agua, biodiversidad, régimen de lluvias y mano de obra. Mientras las investigaciones de estos organismos muestran al sector agropecuario como un gran dinamizador, los planes de desarrollo del país enfatizan y aplican recursos a otros sectores de la economía.
\end{abstract}

\section{Palabras clave}

Desarrollo rural, capacitación, extensión, asistencia técnica rural.

\section{Código de clasificación JEL: I21, J24, Q18}

\begin{abstract}
This article seeks to bring together and expand the discussion on the contradiction between the crisis in number of students admitted to the agricultural faculties in Colombia, from the last decade of the 20 th century, and the potential for food production, expressed by organizations such as FAO, OECD, and table of talks Havana, supported by indicators such as availability of land, abundance of water, biodiversity, rains and labor regime. While investigations of these organisms are the agricultural sector as a great motivator, the country's development plans emphasize and apply resources to other sectors of the economy.
\end{abstract}

\section{Keywords}

Rural development, training, extension, rural technical assistance

* Artículo derivado de trabajos de investigación sobre políticas agrícolas en América Latina desde el grupo de investigación en Administración de Empresas Agroindustriales USTAGRI de la Universidad Santo Tomás Seccional Bucaramanga.

1 Especialista en Gerencia Estratégica. Docente Universidad Santo Tomás Seccional Bucaramanga. Grupo de investigación USTAGRI. Correo electrónico: luiseduardo.santospadilla@gmail.com

2 Magíster en Agronegocios. Decano Facultad de Administración de Empresas Agropecuarias. Universidad Santo Tomás Seccional Bucaramanga. Grupo de investigación USTAGRI. Correo electrónico: edwaramirez@ ustabuca.edu.co 


\section{Introducción}

Según el Banco Mundial, Colombia hace parte de la llamada "despensa agrícola" latinoamericana, capaz de ofrecer soluciones reales al incremento de la demanda por alimentos que se vive en la actualidad; afirmación que es compartida por otros organismos internacionales como la FAO, la OCDE, la CEPAL y por las propias instituciones nacionales como el Ministerio de Agricultura y Desarrollo Rural y el Departamento Nacional de Planeación DNP (2015).

Sin embargo, los hechos muestran una realidad muy diferente: pérdida de muchas de las actividades productivas en los últimos años, abandono del campo por parte de la población joven, desempleo, desmotivación, carencia de servicios y falta de estímulos reales para la pequeña producción campesina, columna vertebral de la producción de alimentos en Colombia.

Esta dura realidad vivida en el campo colombiano en los últimos 30 años también ha incidido en otras variables sociales como la educción, la cual se expresa en falta de motivación de los bachilleres por las profesiones agropecuarias al momento de decidir sobre su futuro profesional. Los registros académicos de las universidades con programas agropecuarios han demostrado que el ingreso de estudiantes a las carreras agropecuarias tiene un comportamiento cíclico (USTA, 2011), directamente proporcional al comportamiento del PIB agropecuario nacional; cuando la economía agrícola mejora, el ingreso de estudiantes universitarios a disciplinas agropecuarias se incrementa, en tanto que cuando este disminuye también ocurre lo mismo con el ingreso de estudiantes.

La explicación de este fenómeno parece muy sencilla: optar por una u otra carrera universitaria no es una decisión unilateral del estudiante, pues con relativa frecuencia es compartida entre el estudiante y el padre de familia y, el grado de motivación o desmotivación de los padres de familia por los buenos o malos resultados de su actividad económica influye necesariamente en la decisión tomada por el estudiante.

\section{Breve reseña histórica}

La extensión y la asistencia técnica agropecuaria se inician en Colombia por parte del Estado en la década de los años cincuenta (Cano, 2003, citado por Cuéllar, 2012), momento en que desplega todo su accionar institucional, técnico, financiero, tecnológico y normativo bajo el modelo proteccionista de producción agropecuaria, es decir, la intervención del Estado en la producción agropecuaria con el fin de garantizar la oferta nacional de las materias primas que requería el país para el desarrollo de su naciente industria, la cual se extiende hasta finales de la década de los noventa, período que corresponde a uno de los peores momentos vividos por la agricultura colombiana en toda su historia. 
Para Guerrero (2014) el fenómeno de pérdida de interés de los estudiantes por las profesiones agropecuarias (administración de empresas agropecuarias, medicina veterinaria, zootecnia, agronomía y otras disciplinas afines) no es casual, sino el resultado de la crisis vivida por la mayoría de las actividades primarias en Colombia, que empieza a evidenciarse a partir de la década del noventa del siglo XX como consecuencia de la aplicación de un modelo de apertura económica que provoca una fuerte contracción del aparato productivo agropecuario nacional.

Según el mismo autor, ya para el 2009 se empieza a identificar un fenómeno de alta subutilización de la tierra apta para el desarrollo de cultivos. La superficie dedicada a actividades agrícolas y silvoagrícolas ascendía, por aquel entonces, solo a 4,9 millones de hectáreas, pese a que se estimaba que 21,5 millones tenían aptitud agrícola; es decir, solo el $22,7 \%$ de la superficie con esa vocación era utilizada para cultivos (Pnud, 2011), situación que ha originado una fuerte reducción en la producción de alimentos y desempleo, tanto de mano de obra no calificada como del talento humano altamente capacitado como lo son los profesionales de las disciplinas anteriormente mencionadas.

Esta especial circunstancia ha venido despertando una fuerte preocupación por parte del Ministerio de Educación Nacional y de la academia en general, hasta el punto de proponer una amplia convocatoria para la generación de estrategias y así aumentar esfuerzos y potenciar estos programas tan necesarios para el desarrollo y la competitividad del país (Mineducación, 2013). La escasa proporción de estudiantes interesados en dedicarse a las carreras agropecuarias en Colombia, ha motivado que gran parte de las más de 50 millones de hectáreas estimadas por el Ministerio de Agricultura para la explotación agrícola y pecuaria, no tengan personal idóneo que se encargue de esas tareas (Castrillón, 2012).

Según la Cooperativa de Veterinarios de Antioquia (COPEVET, 2008, citado por Cabra, 2013), son múltiples los retos a que se enfrenta este grupo de profesiones que sirven al sector agropecuario frente a los cambios económicos, el medio ambiente, la globalización, el crecimiento poblacional, las nuevas exigencias de los mercados, el comportamiento epidemiológico de las enfermedades, entre otros muchos. Para que puedan dar una respuesta nuevas tendencias deben estar preparados nuestros empresarios, profesionales y los Estados, de tal manera que puedan dar una respuesta ágil y eficaz a problemas como la pobreza, el hambre, la desigualdad social, la falta de tierras para la producción de alimentos, falta de tecnologías y sistemas de gestión y de producción agroalimentaria eficientes y sostenibles, el empleo rural y la inclusión social.

Las estadísticas suministradas por el Observatorio Laboral para la Educación Superior (Mineducación, 2013) revelan que desde 2001 y hasta el 2010, de 1.473.608 profesionales titulados en Colombia, solo 23.305, es decir el 1,4\%, eran egresados de carreras afines con la agronomía, veterinaria, zootecnia, entre otras, lo cual evidencia que son pocos los jóvenes que ven en este tipo de ciencias una opción 
viable de cara a su futuro profesional. En general, las titulaciones en educación superior en Colombia siempre han venido creciendo en Colombia, según lo registra el mencionado estudio, a una tasa promedio del $9.0 \%$ anual. No obstante, la dinámica de las profesiones agropecuarias ha mostrado un comportamiento totalmente diferente, llegando a registrar en el 2012 un crecimiento negativo $(-1.0 \%)$ con respecto al año inmediatamente anterior, situación muy preocupante para un sector del cual depende buena parte del desarrollo económico del país y en el que el mundo en general espera grandes aportes en los años por venir en materia de seguridad alimentaria.

Por el contrario, las titulaciones de nivel tecnológico en gestión de empresas agropecuarias pasaron de 11 en el 2010 a 1892 en el 2012 con un incremento altamente positivo del $17.100 \%$ motivado fundamentalmente por el reporte al Ministerio de Educación Nacional de los tecnólogos en administración de empresas agropecuarias formados por el SENA en toda Colombia, los cuales en el pasado alimentaban las bases de datos del Ministerio del Trabajo y Seguridad Social, siendo este el programa de mayor crecimiento dentro del total de titulaciones en el período analizado. Dentro de la cadena de formación muy seguramente este valioso talento humano deseoso de contribuir al mejoramiento de la competitividad del país, buscará en el inmediato futuro su profesionalización, una vez haya logrado su vinculación laboral, accediendo a programas universitarios y más aún, a posgrados en disciplinas afines a su formación tecnológica. Evidencia de ello, es el hecho de que ya algunos programas académicos universitarios han oficializado convenios de homologación con programas de tecnologías, principalmente del SENA, facilitando de esta manera el tránsito de estos estudiantes hacia el nivel profesional.

Los posgrados en todas las disciplinas académicas fueron los niveles que más aumentaron el número de titulados en el período 2010-2012. Las titulaciones en maestrías para todas las áreas del conocimiento en el 2012 fueron de 8045 títulos otorgados, continuando así la tendencia creciente mostrada en los últimos años y representando un $2,8 \%$ del total de titulaciones en educación superior. De igual manera en las especializaciones se otorgaron 60.048 títulos en el 2012 y aunque presentan una ligera disminución en su tasa de crecimiento atribuible probablemente al incremento de las maestrías, siguen siendo las titulaciones de mayor significado en el período de análisis copando el 19\% de las titulaciones en educción superior. No obstante, en el área de la agronomía, veterinaria y afines, tema de nuestro interés, los títulos otorgados de maestría para el 2012 fueron solo de 152 (0.02\% del total), incluidos 24 títulos obtenidos en el exterior y convalidados en Colombia. Por áreas del conocimiento, la mayor concentración de titulaciones se encuentra en economía, administración, contaduría y afines con un total de 1843 títulos expedidos, incluidas 228 convalidaciones de estudios adelantados en universidades extranjeras.

En relación con el tiempo transcurrido entre el momento del grado y su vinculación laboral, el 92\% de los graduados en los niveles de especialización, maestría y doctorado lo hacen en el término de un año, no así el nivel universitario donde solo el 
$78 \%$ logran hacerlo en este tiempo, continuando también aquí la tendencia de mayor dificultad para la ubicación laboral en las profesiones agropecuarias.

Algunos análisis de expertos académicos registrados en La República (2012), Contexto ganadero (2013) y Unimedios de la Universidad Nacional (2014), concuerdan en considerar que este fenómeno obedece principalmente a factores externos como la falta de políticas sectoriales, problemas sociales del ámbito rural, situación económica en el campo, etc., que están desestimulando el estudio de carreras relacionadas con el agro. Las cifras de baja demanda de programas académicos del área agropecuaria y agroindustrial, son el reflejo de la crisis económica por la que atraviesa el sector rural, que incluyen los efectos internos de la apertura económica, la ausencia de garantías gubernamentales, y los problemas de orden público en las zonas rurales.

Según Juan Camilo Restrepo Salazar (2012) se ofertaron en el año 2012, 330 programas académicos de nivel superior del sector agroindustrial colombiano $(47.0 \%$ universitaria, $11.0 \%$ maestría y $4.0 \%$ doctorado). Señala el ministro de Agricultura por aquel entonces, que de cada 100 estudiantes que se gradúan de carreras profesionales o tecnológicas en Colombia, solo 1,8 provienen de ciencias agropecuarias; por eso la idea es fomentar estas ciencias. "Este es un valioso instrumento para que los jóvenes rurales se queden en el campo y creen empresa", aseguró Restrepo (2012). "El país tiene una necesidad clara de profesionales ligados a estas ciencias, razón por la cual, el Ministerio destinó estos recursos para que jóvenes de nivel Sisbén 1 y 2 apliquen para poder estudiar".

\section{Para Restrepo Salazar (2012)}

suplir una necesidad latente que tiene el país en materia de profesionales en las ciencias agropecuarias, es a lo que le apuesta el Ministerio de Agricultura, gracias a los $\$ 14$ mil millones de pesos que serán destinados en becas para que jóvenes rurales accedan a la educación superior y se enfoquen por este tipo de carreras.

Son amplias y angustiosas las demandas de diferentes sectores de la sociedad acerca de la necesidad de reactivar las actividades primarias como una fuente de ingreso para la población del campo y como una alternativa de aprovechamiento de las oportunidades surgidas de los tratados de libre comercio suscritos por Colombia. Para Uribe et al. (Citados en Cuéllar, 2012), hoy se requiere de un direccionamiento estratégico que permita generar acciones dinamizadoras tendientes a incrementar la productividad, haciendo más competitivo el agro colombiano. Sin embargo, no basta con que la innovación tecnológica en el sector agropecuario tenga la capacidad para contribuir a la reducción de la pobreza en las areas rurales, sino que se considera fundamental la participación del campesino como actor principal de su propio desarrollo, respetando su experiencia y sus saberes (Cuéllar, 2012).

Pese a la relativa pérdida de importancia del sector agropecuario en la generación de empleo a nivel nacional en los últimos años, el análisis comparativo acerca de 
la participación en la generación de empleo muestra al sector agropecuario en el segundo lugar después del comercio y los servicios, con un crecimiento en el 2015 del $16 \%$, lo cual confirma la importancia estratégica que aún mantiene en el crecimiento de la economía nacional.

Figura 1. Participación ocupacional por sectores económicos en Colombia

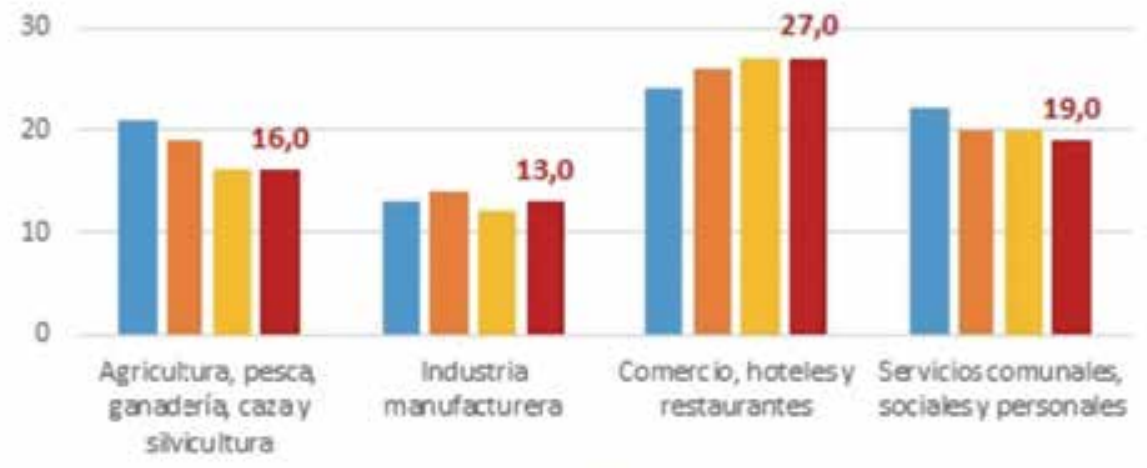

=2005 $=2009=2014$ a 2015

Fuente: Revista Dinero No. 476 (2015).

\section{Un nuevo enfoque de la ruralidad desde el territorio}

En los últimos años el enfoque desde el cual se explica y se trata de abordar la realidad del campo ha dado un viraje pasando de centrar el foco de atención exclusivamente al tema agroproductivo, a dar mayor relevancia al tema espacial del ámbito rural donde se desenvuelven actividades económicas, sociales, ambientales y culturales. Este modelo de desarrollo denominado "Desarrollo rural con enfoque territorial”, según Schejtman y Berdegué (2004) permite integrar la heterogeneidad social de los actores rurales, la multisectorialidad y generación de empleo, la articulación intersectorial, la incorporación de los vínculos urbano-rurales y el papel de la institucionalidad en el desarrollo, con la finalidad de la reducción de la pobreza rural. Es decir, desde la visión de desarrollo rural desde el territorio la cuestión de bienestar y crecimiento va más allá de solo soluciones que vinculen las tierras, cultivos y producción, pues lo rural como categoría del territorio abarca funciones sociales, culturales, gastronómicas, ambientales y forestales, y por supuesto económicas que incluyen además actividades comerciales, de industria rural y de servicios.

Este modelo de desarrollo amplía la visión de lo rural, dando así cabida a otras manifestaciones teóricas y conceptuales de lo que se entiende por campo, y rural como lo contrario a lo urbano o la ciudad (Lozano, 2004). Debido a los constantes cambios 
producidos por la globalización y el advenimiento de la sociedad del conocimiento, el ámbito rural y su principal actividad, la agroproductiva, se han visto golpeados y altamente impactados negativamente como resultado de las brechas existentes entre las oportunidades recibidas en el campo y los beneficios percibidos en la ciudad. Es allí precisamente, como menciona el IICA (2000) donde se hace necesario pensar una nueva ruralidad desde la cual sea posible vincular los temas de producción con los de seguridad alimentaria, combatir la pobreza y buscar la equidad, preservar el territorio y sus valores, cultura y creencias, aumentar los niveles de participación y desarrollo social, así como, incluir a los jóvenes, las mujeres y demás comunidades.

Desde esta visión la agricultura como actividad productiva en el territorio rural, adquiere un carácter multifuncional, integrado por una función ambiental representando en los suelos, la selva y protección de la biodiversidad rural; una función económica que abarca el papel de la agricultura como fuente de ingresos, empleo, bienestar y desarrollo a partir del mantenimiento y producción de sistemas agroproductivos; y una función social, que posee un conjunto de creencias, valores y culturas (FAO, 1999).

El enfoque de desarrollo efectuado en los últimos años en el campo colombiano se ha caracterizado por el desconocimiento y desentendimiento de la realidad rural del país, centrando todos los recursos y esfuerzos en el logro de la productividad agraria, dejando de lado los verdaderos problemas estructurales por los cuales la competitividad y el desarrollo social no han sido posibles. Absalón Machado (2011) sostiene que los impactos de este modelo han desencadenado la inequidad y la exclusión rural al igual que una innumerable cadena de conflictos por la tierra, que ha propiciado diferencias entre los diversos actores de las zonas rurales y la destrucción de los recursos naturales. Pues no en vano, los habitantes rurales del país han tenido que vivir en todos estos años con dos fenómenos particulares: el conflicto armado y el narcotráfico.

Abordar la realidad rural implica adoptar un modelo de desarrollo rural que parta de reconocer las manifestaciones económicas, sociales y ambientales del territorio; dar participación a los diversos actores sociales, mantener un equilibrio entre la producción y el manejo eficiente de los recursos naturales, y generar espacios de participación y representación social.

\section{El acuerdo de paz de La Habana y el sector agropecuario colombiano}

Es importante reconocer aquí la existencia de algunos de los principios orientadores del Acuerdo de Paz de La Habana, en relación con el desarrollo del campo y, particularmente, el papel de la formación profesional, la innovación y el desarrollo tecnológico si se desea comprender mejor las exigencias que plantea una verdadera extensión y asistencia técnica rural integral, tal como lo requiere el sector, y el reto para el Congreso de la República de producir las leyes que hagan posible los cambios 
que demanda el sector agropecuario nacional, para lo cual se torna indispensable contar con profesionales de las ciencias agropecuarias debidamente formados en esta complejidad para que puedan desplegar su bagaje de conocimientos y competencias en pro de los pequeños y medianos productores nacionales y contribuir efectivamente al crecimiento sostenible de nuestro país:

¿Pero, de qué principios estamos hablando específicamente? Se citan dos (2) de ellos:

1. Que el desarrollo rural integral es determinante para impulsar la integración de las regiones y el desarrollo social y económico equitativo.

2. Que en su visión, la Reforma Rural Integral RRI reconoce el papel fundamental de la economía campesina, familiar y comunitaria en el desarrollo del campo, la erradicación del hambre, la generación de empleo e ingresos, la dignificación y formalización del trabajo, la producción de alimentos $\mathrm{y}$, en general, en el desarrollo de la nación, en coexistencia y articulación complementaria Para ello es necesario el reconocimiento y apoyo a las Zonas de Reserva Campesina (ZRC) y demás formas de asociatividad solidaria $\mathrm{u}$ otras formas de producción agraria.

De otra parte, señala el acuerdo, refiriéndose al acceso integral, que en desarrollo de los principios de bienestar y buen vivir, y de integralidad, además del acceso a tierra, el Gobierno Nacional pondrá a disposición de los hombres y mujeres beneficiarios del Fondo de Tierras, planes de acompañamiento en vivienda, asistencia técnica, capacitación, adecuación de tierras y recuperación de suelos donde sea necesario, proyectos productivos, comercialización y acceso a medios de producción que permitan agregar valor, entre otros, y escalará la provisión de bienes públicos en el marco de los Programas de Desarrollo con Enfoque Territorial - PDET (Mesa de Conversaciones de La Habana, 2016):

- El fortalecimiento y la promoción de la investigación, la innovación y el desarrollo científico y tecnológico para el sector agropecuario, en áreas como agroecología, biotecnología, suelos, entre otras.

- Incremento progresivo de los cupos técnicos, tecnológicos y universitarios en las zonas rurales, con acceso equitativo para hombres y mujeres, incluyendo personas en condición de discapacidad. Se tomarán medidas especiales para incentivar el acceso y permanencia de las mujeres rurales.

- Promover la ampliación de oferta y la capacitación técnica, tecnológica y universitaria en áreas relacionadas con el desarrollo rural.

- Asistencia técnica: con el propósito de fortalecer las capacidades productivas de la economía campesina, familiar y comunitaria para desarrollar sus proyectos productivos y estimular procesos de innovación tecnológica, el Gobierno Nacional diseñará e implementará un plan nacional de asistencia integral técnica, tecnológica y de impulso a la investigación. 
Tratándose de un acuerdo de obligatorio cumplimiento para las partes firmantes, esto es Gobierno Nacional y las FARC-EP, solo resta esperar con optimismo la reglamentación por parte del Congreso de la República de los planes nacionales acordados, por ejemplo en temas como la Asistencia Técnica, en los términos que hemos venido mencionando para su pronta aplicación. Un reto de tal dimensión y complejidad solo puede ser visto como una excelente oportunidad para introducir los cambios que el sector agropecuario y en especial la economía campesina han venido esperando a lo largo de las tres últimas décadas. Los profesionales de disciplinas agropecuarias podrán encontrar en este nuevo propósito la oportunidad para poner su talento y competencias al servicio de los intereses del crecimiento competitivo y sostenible del sector agropecuario nacional.

La nueva concepción de ruralidad incluye una visión mucho más amplia de la extensión y la asistencia técnica, capaz de superar el reduccionismo técnicocientífico del pasado, reconociendo su multidimensionalidad en los ámbitos antroposocial, político-institucional, ambiental y económico de la actividad rural, con un fuerte énfasis en los componentes de comercialización y conocimiento de los mercados, tanto nacionales como de exportación, y la gestión empresarial del proceso productivo.

Según Méndez (2006) concebir la producción en sentido amplio, incorporar "tecnologías blandas" (nombre con el que se reconocen los conocimientos y prácticas asociadas a la comercialización, el mercadeo, la administración y la gestión empresarial), resulta altamente necesario, cuando de asegurar el éxito económico se trata. Lo anterior sugiere superar el sesgo técnico-científico de la extensión rural. Esto es, ser conscientes de que poseer una fuerte formación en aspectos asociados a la actividad productiva directa (fitotecnia y zootecnia) no es suficiente para dar cuenta de la totalidad del proceso. En este sentido, disciplinas vistas como complementarias o accesorias durante el proceso de formación profesional, ahora pasan a ocupar un lugar privilegiado.

\section{Hacia un nuevo perfil del extensionista rural}

Tomando en consideración lo expresado por Méndez (2006), es evidente la importancia que entraña la redefinición del perfil del extensionista que hoy se requiere para el desarrollo del campo colombiano y los retos que representa para la academia nacional contar con currículos, metodologías y escenarios de prácticas ajustados a esta realidad para la formación de los nuevos agentes de desarrollo que han de asumir estos retos en el inmediato futuro.

Con este propósito el autor referenciado ha elaborado una síntesis del perfil del actual extensionista rural, centrado esencialmente en asumir una nueva concepción de ruralidad con un enfoque multidimensional, el análisis y reconocimiento de los diversos contextos internos y externos de la actividad rural, la valoración del rol de 
la agricultura como dinamizador del desarrollo nacional, asumir la actividad con enfoque de cadenas productivas, fomentar la participación campesina en actividades productivas a través de formas de economía solidaria y, finalmente, formar los cuadros directivos de las formas asociativas para que puedan realizar una gestión empresarial eficiente y sostenible, entre otros.

También Parra (2012), refiriéndose al papel de la educación para una nueva ruralidad resalta la importancia de incluir en los currículos de la educación superior, además de los aspectos tecnológicos del desarrollo agropecuario, el desarrollo de competencias que posibiliten una mejor comprensión de la realidad circundante, una mayor capacidad para argumentar y motivar los cambios requeridos y para proponer soluciones que respondan a las condiciones de la gente y su territorio:

Si bien el desarrollo de la economía en los territorios rurales depende del mejoramiento tecnológico, existen otras variables indispensables que tienen que ver con el desempeño del individuo; son saberes, aptitudes y actitudes que le permiten al campesino agricultor o al empresario rural tomar las más adecuadas decisiones para lograr éxito en su actividad. El conocimiento, el criterio y las destrezas que se requieren para lograr objetivos, tienen que ver con capacidades para conocer e interpretar la realidad, para comunicarse con los demás, para tomar decisiones, para buscar información, para trabajar en equipo, en fin: saber, saber hacer y saber actuar en contexto, para lograr resultados. Esto es lo que se puede entender como capacidad gestora.

Attman, citado por Parra (2012) manifiesta que "hoy los profesionales presentan debilidades que se traducen en desconocimiento de la complejidad rural, segmentación disciplinaria en su análisis, desvalorización de las formas de vida en el campo y desconocimiento de modelos de intervención en las distintas comunidades rurales", lo cual se convierte en un importante reto para la estructuración de currículos de los programas dirigidos a la formación de tecnólogos y profesionales para el campo.

Ya desde 1998 Chaparro (1998) había puesto de manifiesto la necesidad de enriquecer la formación basada especialmente en la información y dar el paso que promueva la construcción de la sociedad del conocimiento y del aprendizaje, “...es decir una sociedad con capacidad para generar conocimiento sobre su realidad y su entorno, y con capacidad para utilizar dicho conocimiento en el proceso de concebir, forjar y construir su futuro" (Chaparro, 1998).

\section{Recomendaciones de política agropecuaria de la OCDE para Colombia 2015}

A raíz de la solicitud formal de Colombia para hacer parte de los países miembros de la OCDE en el 2013, este organismo ha venido evaluando periódicamente las políticas agropecuarias adoptadas por Colombia y formulando algunas recomendaciones que deben ser tenidas en cuenta para el logro de este propósito.

Entre ellas se encuentran: 
Para lograr su objetivo de crecimiento sostenible agrícola y superar los desafíos estructurales, Colombia debe potenciar políticas que respalden su competitividad a largo plazo. Los recursos públicos deben destinarse a eliminar las importantes deficiencias existentes en el sistema de tenencia de la tierra, infraestructura, gestión del agua y del suelo, sistemas de inocuidad alimentaria y de salud animal y vegetal, infraestructura de transporte, sistemas de información de mercado, educación, investigación y desarrollo, servicios de extensión, asistencia técnica, entre otros. Asimismo, el ordenamiento institucional es débil, tanto a nivel departamental como municipal, lo cual exige mejoras en la gobernanza y en la coordinación de la política agrícola. Colombia también ha suscrito e implantado recientemente varios tratados de libre comercio con importantes socios comerciales, en virtud de los cuales se ha comprometido a eliminar gradualmente una amplia gama de medidas en frontera para productos agropecuarios.

Dice la OCDE (2015) "El apoyo a la agricultura debe centrarse en las reformas estructurales a largo plazo", en actividades como:

- Redirigir los esfuerzos de las políticas en las inversiones estratégicas que actualmente cuentan con apoyos insuficientes, como es el caso de bienes públicos.

- Incrementar la inversión en infraestructuras de riego y mejorar la supervisión regulatoria sobre el abastecimiento, uso y almacenamiento del agua. Aumentar la inversión en infraestructura de transporte.

- Es necesario implantar en Colombia una política inclusiva de acceso a la tierra, a pesar de la complejidad política existente, para poder estabilizar el país y promover el desarrollo rural.

- Actualizar el sistema catastral. Acelerar el registro de los derechos sobre la tierra.

- Reforzar y mejorar el sistema de tributación de la tierra. Estas medidas podrían complementarse con una evaluación del sistema actual de valoración de la tierra y de los procedimientos para la adquisición y transferencia de tierras.

Colombia, en concepto del Banco Mundial, hace parte de la llamada "despensa agrícola latinoamericana", que puede ofrecer soluciones reales al incremento de la demanda mundial por alimentos que se vive en la actualidad. A pesar de esto, el sector agrícola en Colombia está en crisis, trayendo como consecuencia la quiebra de los pequeños y medianos agricultores aumentando las cifras de pobres en el país, que según cifras del Banco Mundial, la población rural que vive por debajo de la línea de pobreza rural nacional en Colombia es del 46,8 \% (Banco Mundial, 2013, 2014).

La política de Transformación productiva del macrosector agro, impulsada por el Gobierno colombiano (Minagricultura, 2012), ha puesto como el centro de su actividad la articulación entre actores privados y públicos para alcanzar los resultados 
estratégicos de mejoramiento de la competitividad y productividad sectorial con una visión amplia del desarrollo sostenible que incluye lo económico, lo social y lo ambiental de seis sectores básicos de la economía agraria nacional como lo son:

- Chocolatería, confitería y materias primas

- Palma, aceites, grasas vegetales y biocombustibles

- Camaronicultura

- Lácteos

- Hortofrutícola

- Carne bovina

No obstante, después de cuatro (4) años de su implementación, los resultados en materia de desarrollo sostenible del campo colombiano son bastante precarios y lo son peor aún para el caso de la producción de alimentos por parte de la economía campesina, que parece excluida de tal iniciativa. La reforma y el fortalecimiento de la institucionalidad agropecuaria, como elementos esenciales de la política para el sector pasa por el mejoramiento de la presencia de dichas instituciones en el nivel local, con personal idóneo, recursos suficientes de toda índole y alejados de la politiquería local.

\section{El Plan Nacional de Desarrollo 2014-2018 y el sector agropecuario}

Existe un expreso reconocimiento del Estado colombiano, tanto de la importancia del sector agropecuario para el desarrollo del país, así como de las condiciones de atraso actualmente imperantes, sin embargo, al momento de concretar dicha política en programas y proyectos no hay una asignación de recursos ni un liderazgo de las agencias del Estado que muestren real interés para hacer los cambios requeridos.

Afirma el Plan Nacional de Desarrollo de Colombia 2014-2018: En cuanto al enfoque rural, las brechas entre el campo y la ciudad en materia de indicadores sociales son demasiado grandes, pues en el campo se concentran los principales problemas de pobreza, falta de educación y falta de infraestructura, entre otros. Además, muchos de los problemas de violencia y narcotráfico están concentrados en la ruralidad. Si Colombia quiere consolidar la paz en su territorio, debe redoblar su atención al campo colombiano. Por eso es necesaria una estrategia integral de transformación del campo, que lo atienda y lo modernice como es debido, comenzando por lo más básico, como tener cartografía, catastro, títulos de propiedad y vías de acceso. Esa estrategia de transformación del campo tiene anexa la estrategia de crecimiento verde, porque la sostenibilidad del crecimiento económico depende también de los aspectos ambientales. Hay una gran complementariedad entre las estrategias de transformación del campo y crecimiento verde, porque la mayoría de 
metas ambientales está asociada con la conservación y explotación sostenible de nuestros recursos naturales.

No hay duda de que la construcción de la paz le permitirá al país potenciar su desarrollo económico a través de ambientes más seguros y atractivos para la inversión, con una mejor y más equitativa distribución de las oportunidades, y una adecuada atención a las prioridades en temas fundamentales como la reducción de la pobreza y la inequidad, y la provisión de servicios sociales esenciales, como la educación y la salud, a lo largo del territorio (Plan Nacional de Desarrollo 2014-2018).

\section{Garantizar el bienestar de las comunidades rurales}

El bienestar de las comunidades rurales debe ser uno de los enfoques fundamentales de la política pública, mediante estrategias como el desarrollo rural integral que busquen corregir los desequilibrios regionales en la provisión de bienes públicos y servicios sociales, promover la igualdad de oportunidades para los pobladores rurales y el aumento significativo de la competitividad sectorial sobre la base de la economía campesina como principal fuente de ingresos en las áreas rurales, especialmente en territorios con alto riesgo de conflictividad social y económica.

El desarrollo rural integral requiere además de estrategias para facilitar la comunicación entre las personas, acceder a la información de servicios como la salud, justicia y educación o mejorar la productividad de los negocios, a través de tecnologías de información y de políticas para promover el acceso, el uso y su incorporación en la vida diaria de las personas.

\section{Conclusiones}

La implementación del Acuerdo de La Habana en materia agropecuaria (24/08/2016) "Hacia un nuevo campo colombiano: Reforma Rural Integral”, con el cual se busca sentar las bases para la transformación del campo, y crear condiciones de bienestar para la población rural, tiene en la Extensión y Asistencia Técnica un punto de apoyo fundamental, como quiera que de la calidad de la asistencia técnica que se preste a los pequeños y medianos productores depende buena parte del éxito en materia de rendimientos y rentabilidad del negocio agrícola.

La implementación de estos acuerdos en el Congreso de la República, proceso actualmente en marcha, deberá reflejar una clara política de Estado y una normatividad que permita el logro de las transformaciones que requiere el sector agropecuario para ponerse a tono con la dinámica y con las expectativas del mundo contemporáneo en materia de seguridad alimentaria. Necesariamente este nuevo escenario requiere de compromisos de actuación de amplios sectores de la sociedad, entre ellos la academia. Probablemente los gremios de la producción, tanto nacionales 
como la inversión extranjera encontrarán en el sector agropecuario una excelente oportunidad de inversión una vez superado el escollo del fenómeno de violencia en el campo, buscando aprovechar las oportunidades que brindan los tratados de libre comercio suscritos por Colombia.

Un reto de tales dimensiones exige un nuevo perfil del extensionista, capaz de ofrecer soluciones a la integralidad de la problemática campesina, es decir, contar con una visión transdisciplinar que supere el sesgo esencialmente productivista de la asistencia técnica y tecnológica del pasado y que exista la garantía de la provisión de servicios, tales como los avance en aspectos técnico-productivos, organizativos y sociales, de gestión, administración. Informática, finanzas, mercadeo y capacitación.

También la academia tiene un importantísimo rol que cumplir para contribuir al logro de este noble propósito, el cual debe extenderse hacia dos campos bien diferenciados, a saber: la actualización de los profesionales actualmente vinculados a estas actividades y segundo, la formación de nuevos profesionales con currículos ajustados a esta nueva realidad.

\section{Referencias}

Amtman, C.A. (2000). La formación profesional para el desarrollo rural en América Latina. En Memorias del Seminario Internacional "La Nueva Ruralidad en América Latina”. Bogotá. Red de Bibliotecas Virtuales de Ciencias Sociales de América Latina y el Caribe - Red de centros miembros de Clacso. Recuperado de http://www.clacso.org.ar/biblioteca biblioteca@clacso.edu.ar

Banco Mundial. (2013, febrero 28). Tasa de incidencia de la pobreza, sobre la base de la línea de pobreza rural (\% de la población rural). Recuperado de http://datos. bancomundial.org/indicador/SI.POV.RUHC/countries

Banco Mundial. (2014). Towards sustainable peace, poverty eradication, and shared prosperity. (Hacia la paz sostenible, la erradicación de la pobreza y la prosperidad compartida). Número del informe ACS10900.

Bardhan, P. (2001). Institucionalidad, reformas y desempeño de la agricultura. En Kostas Stamoulis (ed.). Alimentación, agricultura y desarrollo agrícola. FAO, Roma.

Cabra, C.A. (2013). Retos que deben enfrentar las profesiones del sector agropecuario. Revista Journal of Agriculture and Animal Sciences. Medellín, Colombia: Corporación Lassallista.

Cano, J. (2012). Perspectivas de la extensión para la agricultura: multifuncional y a la medida. Conversatorios sobre nuevas tendencias de la Extensión rural en Colombia. Bogotá, D.C.: SENA. 
Cano, J. (2012). La prestación de los servicios de asistencia técnica y transferencia de tecnología desde los gobiernos locales. Guatemala: FODEPAL.

Castrillón, D. (2012, mayo). Carreras agropecuarias, cada vez despiertan menos interés. Revista electrónica CONtextoganadero. Fedegán. Bogotá, Colombia.

Chaparro, F. (1998). Conocimiento, innovación y construcción de sociedad: Una agenda para la Colombia del siglo XXI. Colciencias. Bogotá. 1998. Recuperado de http://hdl.handle.net/11146/728

Contexto Ganadero. (2013). Carreras agropecuarias, cada vez despiertan menos interés. Recuperado de http://contextoganadero.com/reportaje/carrerasagropecuarias-cada-vez-despiertan-menos-interes

Cuéllar, Y. (2012). La innovación como apuesta para el desarrollo de la población rural en Colombia. FACCEA 2(1). Universidad de la Amazonia.

DNP. (2015). Bases del Plan Nacional de Desarrollo de Colombia 2014-2018. Todos por un nuevo país. Bogotá D. C.

FAO. (1999). El carácter multifuncional de la agricultura y la tierra.

FAO. (2015). Agricultura Mundial: hacia los años 2015/2030. Roma.

Guerrero, E., Rojas, A., Torres, M., Bourdon, N. (2014). Plan prospectivo para el desarrollo agrario en las regiones colombianas a partir del posconflicto al año 2025. Revista Ágora, 14(2). Medellín, Colombia: Universidad San Buenaventura.

Instituto Interamericano de Cooperación para la Agricultura IICA. (2000). Nueva ruralidad. San José, Costa Rica.

La República. (2012). Decrece el interés por estudiar carreras del agro. Recuperado de http://www.larepublica.co/agronegocios/decrece-el-inter\%C3\%A9spor-estudiar-carreras-del-agro_28060

Lozano, R. (2004). Aproximación conceptual a la nueva ruralidad, una tarea para comprender su estado del arte. Revista Integración Empresarial, (3), 14-22. USTA Bucaramanga.

Machado, A. (dir). (2011). Colombia rural Razones para la esperanza. Informe Nacional de Desarrollo Humano. Colombia: PNUD.

Mesa de Conversaciones La Habana. (2013, julio 21). Primer informe conjunto de la Mesa de Negociaciones entre el Gobierno de Colombia y las Fuerzas Armadas Revolucionarias de Colombia Ejército del Pueblo. Obtenido de https://www. mesadeconversaciones.com.co

Mesa de Conversaciones La Habana. (2016). Política de desarrollo agrario integral. Borrador Conjunto. Recuperado de https://www.mesadeconversaciones.com.co 
Mineducación. (2013). Resultados de las condiciones laborales de los graduados de educación superior 2001-2012. Observatorio Laboral de la Educación. Graduados en Educación Superior por áreas del conocimiento. Recuperado de hhtp://www. graduadoscolombia.edu.co/html/1732/w3-article-195063.html

Méndez J. (2006). Los retos de la extensión ante una nueva y cambiante noción de lo rural Revista Facultad Nacional de Agronomía, Medellín, 59(2), 3407-3423.

OCDE. (2015). Revisión de la OCDE de las Políticas Agropecuarias de Colombia 2015. Evaluación y recomendaciones de política. Review of Agricultural Policies: Colombia 2015.

Parra, J. (2012). Educación para una nueva ruralidad. Corporación Latinoamericana Misión Rural.

PNUD. (2011). Colombia rural: Razones para la esperanza. Recuperado de http:// planipolis.iiep.unesco.org/upload/Colombia/Colombia_NHDR_2011_resumen. pdf

Schejtman, A., y Berdegué, J. (2004). Desarrollo territorial rural. Centro Latinoamericano para el Desarrollo Rural RIMISP. Santiago de Chile.

Unimedios Universidad Nacional. (2014). Solo 6.6 \% de los bachilleres estudian carreras del agro. Recuperado de: http://agenciadenoticias.unal.edu.co/detalle/ article/solo-66-de-los-bachilleres-estudian-carreras-del-agro.html

Uribe, C., Bernal, G., Contreras, C., Castellanos, O. (2003). Sembrando innovación para la competitividad del sector agropecuario colombiano. Ministerio de Agricultura y Desarrollo Rural. Bogotá. D. C., Colombia. 